\title{
Nuevos aportes para la \\ historia del pensamiento \\ colombiano durante el \\ siglo Xx
}

Sergio Andrés Salgado Pabón

Pontificia Universidad Javeriana,

Bogotá, Colombia.

ssalgado@javeriana.edu.co

Continuando con los tomos aparecidos en 2007 y 2008, el Instituto de Estudios Sociales y Culturales PENSAR de la Pontificia Universidad Javeriana (Bogotá, Colombia) publica una entrega más de la serie Pensamiento colombiano del siglo $X X$, cuyo objetivo es presentar un análisis crítico de las cien figuras más relevantes de la historia intelectual y cultural de este país durante ese siglo. Bajo el cuidado de Carmen Millán de Benavides, Santiago Castro-Gómez y Guillermo Hoyos Vásquez (filósofo colombiano fallecido durante el proceso de edición del libro y a cuya memoria está dedicado), este tercer tomo (de cinco proyectados) analiza diversos pensadores colombianos sin restringir la categoría "pensamiento", como se afirma desde el primer tomo, a "los productos intelectuales de los filósofos y los científicos sociales", y entendiéndola, antes bien, como algo que "abarca también la actividad cognoscitiva de artistas, literatos y activistas políticos que tuvieron alguna incidencia en el devenir nacional durante el transcurso del siglo XX".1

Confiados en haber superado los desaciertos de los tomos anteriores (a la escucha de las reseñas hechas sobre el primer tomo, el prólogo del se-

Guillermo Hoyos Vásquez, "Prólogo", Santiago Castro-Gómez, Alberto Flórez-Malagón, Guillermo Hoyos Vásquez y Carmen Millán de Benavides, Pensamiento colombiano del siglo XX, tomo 1, Bogotá, Pontificia Universidad Javeriana, 2007, p. 9. 
gundo refería ya la necesidad, entorpecida en parte por la estructura misma del proyecto, de atender más a la "producción social del conocimiento" que a figuras individuales convertidas, así, en protagónicas), ${ }^{2}$ y siguiendo, como se proyectó desde el primer tomo, una estructura tripartita para cada texto (biografía, análisis de la producción intelectual de la figura objeto y bibliografía). Estas veinte entradas, escritas por reconocidos especialistas, analizan diferentes figuras pertenecientes a diversos momentos y campos de la historia de Colombia durante el siglo xx, haciendo evidente que "aun hoy, en medio de la información instantánea posible gracias a la Internet, es necesario recuperar vidas y trabajos que son invisibles o son tratados en forma sumaria en la nube informática" (p. 9).

A continuación brindaremos un análisis sucinto de estas veinte entradas deteniéndonos, sin embargo, en aquéllas que, según consideramos, merecen mayor atención por tratarse de figuras esenciales poco conocidas o por exigir observaciones sobre el análisis. De todas se dará cuenta, de cualquier forma, a través de breves agrupaciones que, a pesar de responder más a objetivos pedagógicos que estrictamente analíticos, permitirá aproximarnos a un muy variado abanico de figuras, pues en muchas de ellas los campos, como afirmaría Óscar Torres Duque acerca de Darío Achury Valenzuela, "tienen que ponerse en crisis, sin embargo, para empezar a ser considerados como contextos históricos -o simplemente "campos" en el sentido de Bourdieu-" (p. 165).

La lectura de este tercer tomo nos acerca con un grupo de figuras que podemos agrupar como pertenecientes al terreno literario y que representan una clara mayoría al interior del libro (once de las veinte estudiadas en el mismo).

Analizando los aportes del joven Luis Tejada (1898-1924), cronista perteneciente a la llamada generación de Los Nuevos y al referirse a la Colombia de 1922, llegó a sentenciar: "Este país es esencialmente conservador en todos los aspectos de su vida, pero singularmente en lo que se refiere a la literatura". ${ }^{3}$ Gilberto Loaiza Cano puntualiza los elementos renovadores de quien se preocupara más que ninguna otra figura de su generación por hacer un llamado a la revolución, tanto en el terreno de las

\footnotetext{
2 Guillermo Hoyos Vásquez, Carmen Millán de Benavides y Santiago Castro-Gómez, "Prólogo", Guillermo Hoyos Vásquez, Carmen Millán de Benavides y Santiago CastroGómez, Pensamiento colombiano del siglo xx, tomo 2, Bogotá, Pontificia Universidad Javeriana, 2008, p. 10. Luis Tejada, “Un poeta extraordinario", Luis Vidales, Suenan Timbres, Bogotá, Instituto Colombiano de Cultura, 1976, p. 13. La edición del libro de Vidales, bajo el cuidado de Juan Gustavo Cobo Borda, no indica la fecha exacta de publicación.
} 
letras como en el de la política, pues su impulso renovador y sus intereses lo llevaron pronto hacia el socialismo; y Bruno Mazzoldi quien en un texto de escritura nada ortodoxa esboza y se hace eco de la renovación del poeta León de Greiff (1895-1976) durante la misma década. Con estas dos figuras y la del poeta Luis Vidales (1904-1990), analizada en el segundo tomo de la serie por Javier González Luna, se completa el análisis del trío que reunió las apuestas más feroces de la generación de Los Nuevos en la década de 1920 desde el terreno de las letras. ${ }^{4}$

De igual forma, en este tercer tomo podemos encontrar análisis de reconocidos escritores como Germán Espinosa (1938-2007), Álvaro Cepeda Samudio (1926-1972), Manuel Zapata Olivella (1920-2004), Andrés Caicedo (1951-1977) y a una de las únicas dos mujeres estudiadas en el volumen: Matilde Espinosa (1911-2008), figuras analizadas, respectivamente, por Cristo Rafael Figueroa Sánchez, Andrés Vergara Aguirre, Mara Viveros Vigoya, Carlos-Enrique Ruiz y Edwin Carvajal Córdoba.

De entre estos intelectuales quizás llame la atención encontrarnos con Caicedo, joven y precoz escritor embebido de teatro, cine, Richie Ray, Beatles y Rolling Stones que aún fascina a los jóvenes, y de quien el estudio afirma efectuar diversas puntualizaciones dada la cantidad de inexactitudes "que a lo largo de estos treinta y cuatro años desde su muerte se han ido generalizando en el país hasta crear una imagen no muy precisa o afortunada de lo que representa su vida y su obra en el panorama literario nacional" (p. 105).

Además, este tomo se encarga de rescatar otras figuras poco tenidas en cuenta por la historiografía debido a que tocan diversos campos -ninguno de los cuales se ha encargado de acoger y emprender su estudio con rigor-: Darío Achury Valenzuela (1906-1999), Ernesto Volkening (19081982), Hernando Valencia Goelkel (1928-2004) y Hernando Téllez (19081966), analizados, respectivamente, por Óscar Torres Duque, Santiago Mutis Durán, Felipe Restrepo David y Alberto Bejarano.

El texto de Torres Duque subraya la amplia labor de Achury Valenzuela, que resume con el binomio "escritor y gestor cultural" (Achury

4

La libertad del texto sobre León de Greiffexige exige algunas precisiones. Mazzoldi no trata el tema de la generación de Los Nuevos ni señala (como tampoco lo ha hecho el grueso de la historiografía sobre el tema) que antes de formar parte de ésta, León de Greiff había sido proclamado como perteneciente a la Generación del Centenario (Luis Eduardo Nieto Caballero, Colombia Joven, Bogotá, Arboleda \& Valencia, 1918, pp. 5153). Su desplazamiento hacia la generación de Los Nuevos comienza con su participación en Voz de la Juventud (1917-1919) y Revista Azul (1919), y queda consagrada con su colaboración en Universidad (1921-1922) y Los Nuevos (1925). 
Valenzuela tuvo diversos cargos en la administración pública que le permitieron colaborar, por ejemplo, en la creación del Instituto Caro y Cuervo en 1944), para rescatar su interés juvenil en la vanguardia europea y su giro posterior hacia un arte nacional y americano ante su preocupación por una "colonización mental" (p. 171). Mutis Durán rescata del olvido a quien fuera "el primero en Colombia en hacer crítica de cine" (p. 187): Volkening, de quien subraya sus aportes como traductor y editor, entre otras facetas, pero sobre todo como escritor "pues eso es lo que es" (p. 189). David Restrepo estudia a Valencia Goelkel, fundador junto con Jorge Gaitán Durán (1925-1962), de la legendaria revista Mito (1955-1962), para concentrarse en su labor como escritor, traductor y crítico de arte, cine y, sobre todo, literatura, dejando de lado su labor como gestor y editor, igualmente amplia e importante. Comentando brevemente su labor como escritor, Bejarano rescata de Téllez su trabajo como lector, crítico y traductor "a la francesa" y "de literatura francesa", labor no exenta de "aspectos paradójicos" que constituyen, según afirma, "su principal aporte" (p. 361).

Ahora bien, en el campo filosófico podemos encontrar a Abel Naranjo Villegas (1910-1992) y Gerardo Valencia Cano (1917-1972), analizados por Jorge Aurelio Díaz Ardila y Benildo Estupiñán Solís, respectivamente. De Naranjo Villegas, Díaz Ardila rescata haber sido "uno de los pioneros de los estudios filosóficos en Colombia", junto con "Cayetano Betancur, Luis Eduardo Nieto Arteta, Danilo Cruz Vélez, Jaime Vélez Sáenz y Rafael Carrillo", "en una época en que la filosofía se cultivaba casi exclusivamente en los seminarios eclesiásticos, marcada por la doctrina de Tomás de Aquino y como complemento de la formación teológica del clero" (p. 16). Estupiñán Solís subrayará de monseñor Valencia Cano la manera como "su pensamiento y acciones" se inscriben en la línea de la Teología de la Liberación (p. 251).

Escrito a tres manos (por Camilo Leyva, Manuela Ochoa y Juan Carlos Osorio), en este tomo encontramos, además, el análisis de una segunda y última figura femenina: la artista Feliza Bursztyn (1933-1982), precursora de la instalación en Colombia, y el estudio de Germán Arciniegas (1900-1999), quien vivió casi por completo el siglo xx. Para el análisis del americanista colombiano se convoca a quien es conocido como el mayor especialista en su obra: Juan Gustavo Cobo Borda, cuyo análisis realiza un recuento por la extensa vida del americanista en el terreno diplomático. En sus libros, alrededor de 63, se encuentra El estudiante de la mesa redonda, publicado en España en 1932, donde abordó algunas de las polémicas en las que estuvo inserto y en sus publicaciones periódicas. Sin embargo, Cobo Borda olvida, una vez más, la primera publicación perió- 
dica dirigida por Arciniegas: Voz de la Juventud (1917-1919), publicación que diera origen efectivo a su americanismo al exigirle tomar contacto con gran número de núcleos de los movimientos estudiantiles de principios de siglo Xx en el continente (olvido bastante significativo). ${ }^{5}$

Pero la amplitud de campos tocados por este tomo tal vez sea más notoria con la presencia del biólogo Armando Dugand Gnecco (1906-1971), el médico e higienista Jorge Bejarano Martínez (1888-1966), el empresario Ricardo Olano Estrada (1874-1947), cuyo papel y reflexión son resumidos en dos palabras: "urbanismo y civismo" (p. 541); el psicoanalista Simón Brainsky Lerer (1939-2005), en una "Laudatio" expresa (p. 558) que bien pudo haber sido algo más analítica; y el asesinado político conservador Álvaro Gómez Hurtado (1919-1995). Figuras analizadas, respectivamente, por Jairo Solano Alonso, Zandra Pedraza, Fernando Botero Herrera, Ignacio Abello T. y Germán Saavedra Soler.

Esta amplia galería brinda al tercer tomo de la iniciativa del Instituto PENSAR un lugar clave en la difusión y análisis de figuras esenciales para pensar la historia de Colombia durante el siglo xx en diversos campos y momentos. Esperemos la llegada pronta de los siguientes tomos.

5 El especialista en Arciniegas había olvidado Voz de la Juventud. En su recuento de las publicaciones dirigidas por el americanista (Juan Gustavo Cobo Borda, "Las revistas de Arciniegas", La otra literatura latinoamericana, Bogotá, El Áncora-Procultura-Colcultura, 1982, p. 156), data de manera incorrecta al intentar referirla ya sea entre 1919 y 1920 (Juan Gustavo Cobo Borda, "Germán Arciniegas: una visión de América", Una visión de América. La obra de Germán Arciniegas desde la perspectiva de sus contemporáneos. Bogotá, Instituto Caro y Cuervo, 1990, p. XXIV).

Recibido: 13/10/2013. Aceptado: 08/09/2014 